\section{Sizing-up the brain}

SIR - Four points stand out from the recent work of J. P. Rushton on racial differences in brain size (controlling for body weight) that you deemed too problematical for scientific publication ${ }^{1}$.

(1) The brain size/IQ link exists but is slight. (Only one modern study reports a correlation as high as 0.35 (ref. 2), and this involved just one of several possible indices of brain size).

(2) The brain size/race link also exists, but is slighter. (Using thousands of subjects, the Black-White brain size difference approximates a mere one-sixth of a standard deviation in males and onethirtieth s.d. in females.)

(3) The brain size/sex link is substantial. (Males and females differ by roughly a full s.d.)

(4) The IQ/sex link is non-existent as Sir Cyril Burt had recognized by 1912 (The only important sex difference in psychometric intelligence is that the male s.d. is wider by 1 IQ point ${ }^{3}$ - thus the often-talked-of male superiority in 'spatial intelligence' is minute ${ }^{4}$.)

These four propositions are unlikely to be embraced in any single, simple theory - as Rushton and Ankney largely admit in their (now published) reports ${ }^{5,6}$. Moreover, these four propositions cannot attest, help explain, or even be squared with the substantial (1 s.d.) Black-White difference in IQ tests.

Surprisingly, however, Schluter ${ }^{7}$ tries to cast doubt on the brain size/sex link. (Contrary to his apparent intentions, to dispense with this link would remove the biggest current obstacle to any claim that racial differences in intelligence result from differences in brain size; but Schluter is undeterred.) Schluter's argument is flawed. Its key proposition is: "If men truly have larger brains for their body size than women, men should be shorter than women of equal brain weight." Thus Schluter, finding brain-weight matched men still taller than control women, takes the link between brain size and sex to be false. However, men could, of course, on average, both be taller than women and have larger brains than would be expected from their size alone - as evidently happens. Schluter's prediction should have been that, with brain weight matched, the sex difference in height would be reduced, but not, of course, reversed.

Altogether, nothing coherent or of any theoretical significance has been learned so far from Rushton's latest sally into the minefield of psychological race differences. But Nature seems resolved to strive for political correctness and to expect Rushton and others in the field to meet higher academic standards than their critics. At the same time, in the heat of controversy over racial differences, Rushton's 'scientific' critics will sometimes advance arguments that are self-defeating if accepted, but embarrassingly illogical in any case.

Scientific racists, if there are any, can be well pleased that bias and unreason will be deployed against them from high places. To provide such satisfaction is, perhaps, the inevitable cost of declining to examine Rushton's work openmindedly on its scientific merits.

\section{R. Brand}

Department of Psychology,

University of Edinburgh,

Edinburgh EH8 9J2, UK

SIR - Despite statistical problems in the literature, one of which Schluter ${ }^{7}$ notes, there seems to be adequate evidence that women have a mean brain mass about $100 \mathrm{~g}$ less than men of the same height. This statement is based on direct comparisons of mean brain sizes for each sex in the same height group (for example Table 1 of ref. 8).

Correction for body size, within or among mammalian species, as with Jerison's ${ }^{9}$ extra neurons above maintenance, is nevertheless unsure. This is because we don't know the extent (if any) to which larger body size decreases the evolutionary cost of a brain larger than needed for maintenance, as a result of the brain being proportionally smaller. To the extent that such a decrease in cost occurs, allometric correction will be over-correction because allometry itself involves more than the maintenance level. It is therefore conceiveable that larger mammals, among and even within species, are on the average more intelligent than smaller ones with the same departure from the trend line.

However, Haug ${ }^{10}$ showed that neurons are more densely packed in the cerebral cortex of women than of men; the total average number is not detectably different. Neurons are also more densely packed in humans than in other mammals, but larger brains of each human sex have less densely packed neurons than do smaller brains, although still a greater total number ${ }^{10}$. Neuronal branching has not been studied.

Some years ago ${ }^{11}$ I estimated the sexspecific correlation between human brain size and measured intelligence to be 0.3 , from indirect evidence. This has now been corroborated ${ }^{2}$ by direct evidence from a small sample.

Whether there are differences in general or special intelligence as a result of the various aspects of sexual dimorphism in human brains can't be investigated from standard tests, because those questions on which one sex does better are eliminated when the tests are prepared. Biology really isn't destiny, and dimorphism doesn't imply any overall inequality, but (as Haldane $\mathrm{e}^{12}$ realized) understanding their effects is necessary in order to deal with these effects in a rational way.

Leigh M. Van Valen

Department of Ecology and Evolution,

University of Chicago,

Chicago, Illinois, USA

SIR - Some of your correspondents on the race and sex dependence of brain sizes illustrate Aldous Huxley's definition of philosophy: the finding of bad reasons for things you believe for worse reasons. The most compelling reason for believing that the races and sexes have different mental characteristics is the fury that this suggestion arouses in the politically correct.

Every orthodoxy in history, from the Inquisition to Marx to Lysenko, has shown inspired flair in embracing, and seeking to enforce belief in, nonsense. I don't know how they do it, but they are wonderfully reliable. Any claim asserted with menaces by an orthodoxy can safely be taken as false.

\section{David Jones}

Physical Chemistry Department,

The University,

Newcastle upon Tyne, UK

1. Maddox, J. Nature 358, 187 (1992)

2. Witierman, L. et al. Intelligence 15, 223-228 (1991).

Jensen, A. R. Bias in Mental Testing, 627-8, (Methuen, London, 1980)

4.. Wechsler, D. The Measurement and Appraisal of Adult Intelligence, 137 (Williams \& Wilkins, Baltimore. 1958).

5. Rushton, J. P. P. Intelligence 16, 401-413 (1992).

6. Ankney, C. D. Intelligence 16, 329-336 (1992)

7. Schluter, D. Nature 359, 181 (1992).

Chrzanowska, G. \& Krechowiecki, A. Gegenbaurs Morph Jb. 121, 192-208 (1975).

. Jerison, H. J. Hum Biol, 35, 263-291 (1963).

10. Haug. H. Am. J. Anat. 180, 126-142 (1987).

11. Van Valen, L. M. Am. J. Phys. Anthrop. 40, 417-424 (1974)

12. Haldane, J. B. S. The Inequality of Man and Other Essays (Chatto \& Windus, London, 1932).

\title{
Breaking the code
}

SIR - "Daedalus" (Nature 358, 22; 1992) defines a "random sequence", claims that Nature is a nonrandom sequence, and suggests a way to encode an issue of Nature by a terse programme. But let that issue include an article which lists a sequence which is "random" by Daedalus's definition. (Suppose the article is about random sequences and includes examples.) That issue of Nature will then be a random sequence because part of it is. So that issue of Nature itself will be unencodable.

\section{Frank J. Leavitt}

Jakobovits Center for Jewish Medical Ethics,

Ben Gurion University of the Negev, Beer Sheva, Israel 